\title{
Evidências de Validade de Construto da Escala de Componentes do Amor
}

\author{
José Augusto Evangelho Hernandez 1 \\ Universidade do Estado do Rio de Janeiro
}

\begin{abstract}
RESUMO - Este estudo buscou verificar as propriedades psicométricas da versão brasileira da Escala de Componentes do Amor reduzida. Participaram 491 pessoas que estavam em relacionamentos amorosos. Análise Fatorial Exploratória extraiu três fatores que explicaram $73,5 \%$ da variância comum que não reproduziram a estrutura original. Os fatores extraídos apresentaram correlações de moderadas a fortes entre si e com os componentes da Escala Triangular do Amor reduzida. A solução de Schmid-Leiman indicou a existência de fator de segunda ordem na estrutura da escala. Os Alfas revelaram elevados índices de consistência interna para as dimensões do instrumento. Análise Fatorial Exploratória adicional com o conjunto de itens das duas medidas revelou uma estrutura fatorial indicativa de que estes possam representar os mesmos componentes do amor
\end{abstract}

Palavras-chave: psicologia do amor, relacionamentos íntimos, validade, fidedignidade

\section{Evidence of Construct Validity of the Love Components Scale}

\begin{abstract}
This study aimed to evaluate the psychometric properties of the Brazilian version of the reduced Love Components Scale. Participants were 491 people in loving relationships. Exploratory factor analysis extracted three factors that explained $73.5 \%$ of the common variance that did not reproduce the original structure. The factors showed moderate to strong correlations with each other and with the components of the reduced Triangular Love Scale. The Schmid-Leiman solution indicated the existence of a second-order factor. The reliability coefficients revealed a high internal consistency for all dimensions of the instrument. Additional exploratory factor analysis with the set of items of the two measurements revealed structural indications that they may represent the same components of love.
\end{abstract}

Keywords: psychology of love, close relationships, validity, reliability

Nos primórdios da pesquisa em Psicologia do Amor predominaram os estudos sobre a atração interpessoal, conceito que é relacionado com o amor, porém não idêntico. Sob a influência das teorias comportamentalistas, a atração por outra pessoa era vista como resultado de sentir-se gratificado em presença da mesma (de tal modo, capacitando-a a tornar-se um reforçador secundário). Ambos, condicionamento respondente e operante, teriam um papel importante no desenvolvimento da atração interpessoal.

Até os anos 1960 havia a suposição de que as formas fracas de atração interpessoal como gostar e as fortes como o amor romântico eram considerados diferentes pontos de um mesmo contínuo quantitativo de sentimentos positivos. Além disso, havia o pensamento de que os determinantes causais de gostar e amar seriam os mesmos, diferindo apenas em magnitude. As forças causais, que poderiam ser geradas de maneira fraca em laboratório para produzir o gostar, seriam suficientemente intensificadas no mundo real para, eventualmente, produzir o amor romântico. Contudo, essas suposições, de que as mesmas causas produzem o gostar e o amor romântico, foram problematizadas, testadas e reformuladas (Berscheid, 2010).

Rubin (1970, 1973), marco inicial do estudo sistemático do amor, explorou os conceitos de gostar e amar e demonstrou que, ainda que ligados, podem ser independentes. O

1 Endereço para correspondência: Instituto de Psicologia, UERJ, Rua São Francisco Xavier, 524, Sala 10028, Bloco D, Maracanã, Rio de Janeiro, RJ, Brasil. CEP: 20.550-900. E-mail: hernandez.uerj@gmail.com pesquisador identificou como componentes do amor o precisar do outro, os cuidados com o outro e a presença da intimidade, envolvendo confiança e proximidade. Quanto ao gostar, Rubin identificou duas dimensões fundamentais: a afeição e o respeito. Afeição é baseada na experiência do calor emocional e da proximidade da outra pessoa. $\mathrm{O}$ respeito se baseia na admiração pelas características ou ações do outro, trata-se de uma forma de gostar mais calma, mais distante. A partir disso, Rubin criou, por meio de análise fatorial, as escalas do Amor e do Gostar, discriminando os pensamentos, as expectativas, os comportamentos e os sentimentos associados ao amor daqueles referentes ao gostar, e unindo os estudos e concepções teóricas sobre atração interpessoal e amor.

Influenciados pelo trabalho de Rubin (1970), os psicólogos sociais começaram a usar técnicas psicométricas, tais como análise de componentes principais, para desenvolver medidas do amor. Entre estas, encontramos a Escala de Componentes do Amor-Reduzida (ECA-R) de Critelli, Myers e Loss (1986), que foi traduzida para a língua portuguesa. Na presente pesquisa, a estrutura fatorial, a validade convergente e a consistência interna dos itens dessa versão do instrumento foram verificadas a partir dos escores de brasileiros.

Na construção do modelo de amor, Critelli et al. (1986) foram fortemente influenciados pelas teorias de orientações psicodinâmicas e clínicas de Fromm (1956/1966) e Maslow (1968/1974), além das concepções de Rubin (1970). Fromm distinguiu dois tipos de amor: o verdadeiro amor, identificado como uma atividade que se caracteriza por cuidado, responsabilidade, respeito e conhecimento; e o falso 
amor, que é baseado em submissão, passividade e dotado de contornos neuróticos. O amor maduro rompe e supera o sentimento de isolamento, mas permite a preservação da integridade e individualidade própria de cada um. Subjacente ao amor passivo está a simbiose, na qual as pessoas evitam o sentimento de isolamento e separação tornando-se parte do outro, que as dirige e as protege. Essa submissão ao outro implica em dependência e falta de integridade pessoal.

De forma similar, na abordagem de Maslow (1968/1974), o amor é basicamente dependência e necessidade de realização. Ele fez uma distinção entre esses dois tipos possíveis de amor, hierarquicamente dispostos conforme a satisfação das necessidades que uma pessoa vai buscar na outra. As quatro primeiras necessidades humanas (biológicas, de segurança, de amor e pertença e as relativas à autoestima) constituem as deficiências, chamadas necessidades $\mathrm{D}$, e as necessidades humanas avançadas (intelectuais, estéticas e de autorrealização) são relativas ao ser, denominadas necessidades S. O amor D enquadra-se no modelo do amor passional, enquanto que o amor $\mathrm{S}$, motivado pela satisfação de necessidades menos primárias e mais transcendentais, talvez seja algo entre o amor pragmático e o altruísta.

Além disso, Critelli et al. (1986) foram influenciados pelo trabalho de Driscoll, Davis e Lipetz (1972), que desenvolveram um sistema discriminativo entre o amor romântico e o conjugal. O amor romântico reuniria necessidades afiliativas e de dependência, sentimentos de exclusividade e de absorção, atração física, paixão e idealização. Por outro lado, o amor conjugal seria definido como o amor adulto maduro, composto de amizade íntima, verdade, ausência de críticas, admiração, respeito, partilha, conhecimento verdadeiro do outro, lealdade e disposição para se sacrificar pelo parceiro.

No construto de Critelli et al. (1986), o amor possui cinco componentes: Dependência Romântica, Intimidade Comunicativa, Excitação Física, Respeito e Compatibilidade Romântica. A Dependência Romântica é caracterizada pela crença em ideais românticos, com ênfase na importância do relacionamento, no quanto o parceiro é necessário e na exclusividade do amor. A felicidade do indivíduo depende do relacionamento com o parceiro. Esse componente é nitidamente relacionado com o falso amor de Fromm e o amor $\mathrm{D}$ de Maslow, embora também envolva aspectos relativos aos sentimentos de responsabilidade pelo bem-estar do parceiro (amor maduro). Nesse componente, está presente a concepção de Rubin sobre o amor romântico: as necessidades afiliativas e de dependência, a predisposição à ajuda e a exclusividade e absorção pelo parceiro. A Intimidade Comunicativa é o componente que enfatiza o sentimento de ser compreendido, de participar de um relacionamento sólido, havendo comunicação e confiança no parceiro. Esse componente identifica-se com o amor verdadeiro de Fromm e o amor S de Maslow. A Excitação Física, que se refere ao estado romântico e sexualmente excitado, apaixonado e fisicamente atraído pelo outro é, portanto, relacionado com necessidades de ordem primária. O Respeito inclui vários dos itens da escala de gostar de Rubin, enfatizando o parceiro maduro, bem ajustado, recomendável e que usufrui de bom conceito. Por fim, a Compatibilidade Romântica, que é resultado da combinação dos ideais românticos enfatizados na Dependência Romântica e na interação harmoniosa, na compatibilidade de humor, na satisfação total e no contentamento dos parceiros.

Na construção da ECA-R, Critelli et al. (1986) reuniram 63 itens que testaram em uma amostra de 123 casais de namorados. Para o sexo masculino, a idade dos participantes ficou entre 18 e 27 anos $(M=20,2 ; D P=1,4)$, para o sexo feminino, de 18 e 28 anos $(M=19,6 ; D P=1,3)$. O tempo de namoro variou de duas semanas a cinco anos com média de seis meses. Os escores dos respondentes foram submetidos a uma Análise Fatorial para componentes principais com rotação varimax. Numa solução final com 35 itens foram retidos cinco componentes, baseado no critério de Kaiser (1960) que explicaram 42,2\% da variância total: o primeiro, identificado como Dependência Romântica, explicou 25,7\%; o segundo, Intimidade Comunicativa, 6\%; o terceiro, Excitação Física , 3,9\%; o quarto, Respeito, 3,5\% e o quinto, Compatibilidade Romântica, 3,1\%. Alguns itens saturaram em mais de um componente, nesses casos, foram incluídos no componente em que houve a saturação mais forte. Os componentes mostraram correlações moderadas entre si, que variaram de 0,37 a 0,71. Contudo, os autores não cogitaram acerca da possibilidade de uma dimensão geral do amor abrangendo os cinco componentes. Os coeficientes de consistência interna de cada um dos cinco componentes foram elevados e podem ser observados na Tabela 2 .

Carreño e Serrano (1995) examinaram 545 estudantes universitários com uma versão espanhola da ECA-R, replicaram o estudo de Critelli et al. (1986) e encontraram os cinco componentes originais. A variância total explicada foi de $53,7 \%$, os três primeiros componentes extraídos (Intimidade Comunicativa, Dependência Romântica e Excitação Física) agruparam todos os itens determinados com saturações superiores a 0,50, o componente Respeito apresentou cinco itens com saturações superiores a 0,50, mas para os itens da Compatibilidade Romântica as saturações apareceram distribuídas em outros componentes.

Carreño e Serrano (1995) relataram correlações entre os itens e o conjunto da escala que foram superiores ao esperado para subescalas claramente diferenciadas. Também descobriram uma coesão maior entre Intimidade Comunicativa e Dependência Romântica, além de moderadas correlações entre as escalas de componentes $\left(r_{\text {médio }}=0,50\right)$. Os coeficientes de consistência interna (alfas) para a escala total e para cada componente foram elevados, semelhantes ao estudo original (Tabela 2). Segundo esses pesquisadores, esses dados sugerem a existência de uma medida geral do amor mediante cinco elementos diferentes.

Além dos estudos de Critelli et al. (1986) e Carreño e Serrano (1995) não foram encontradas outras publicações no plano internacional. Nas publicações nacionais consta apenas o estudo de Hernandez e Oliveira (2002), que apenas fizeram uma tradução da ECA-R (back translation) para a língua portuguesa e relacionaram essa medida com a satisfação amorosa. As buscas foram realizadas na base de dados PsycINFO. Numa revisão sobre o desenvolvimento de medidas do amor de Hatfield, Bensman e Rapson (2011) identificaram 32 instrumentos em quase sete décadas de investigações. Contudo, os autores admitiram a possibilidade de que algumas medidas não tenham sido localizadas. Com 
efeito, observou-se que a ECA-R de Critelli et al. (1986) não constou da lista dessa pesquisa. Curiosamente, as medidas do amor mais populares (Hatfield \& Sprecher, 1986: Hendrick \& Hendrick, 1986; Sternberg, 1997), conforme Masuda (2003), Graham e Christiansen (2009) e Berscheid (2010), foram publicadas todas no mesmo ano e em importantes periódicos da psicologia internacional, da mesma forma que Critelli et al. (1986).

O presente projeto pretende, por meio dos escores de brasileiros, verificar a estrutura fatorial da versão reduzida da ECA, sua consistência interna e sua relação com outra medida similar testada no Brasil, a Escala Triangular do Amor de Sternberg, na versão reduzida (ETAS-R) de Gouveia, Fonseca, Cavalcanti, Diniz e Dória (2009).

\section{Método}

\section{Participantes}

Foi recrutada uma amostra não probabilística de conveniência com 491 indivíduos do sexo feminino $(n=282$; $57,4 \%)$ e masculino ( $n=209 ; 42,6 \%)$ na cidade de Porto Alegre/RS. Todos os participantes estavam vivenciando um tipo de relacionamento amoroso: casados e união estável $(n=177 ; 36 \%)$, noivos e namorados $(n=296 ; 60,3 \%)$, amantes $(\mathrm{n}=2 ; 0,4 \%)$ e não forneceram essa informação $(n=16 ; 3,3 \%)$. A idade dos respondentes variou de 18 a 63 anos, com média de 29,2 anos e desvio padrão de 9,3 anos. O tempo de duração da relação amorosa variou de um mês a 30 anos, com média de 6,4 anos e desvio padrão de 6,9 anos. Cento e quinze $(23,4 \%)$ pessoas informaram que têm filho na relação e 357 (72,7\%) não, 19 (3,9\%) não forneceram esse dado. Cento e oitenta e um (37,5\%) declararam coabitar com o parceiro e $289(58,9 \%)$ não, 18 (3,7\%) não forneceram essa informação. A escolaridade dos participantes variou de ensino fundamental $(n=8 ; 1,6 \%)$, ensino médio $(n=33$; $6,7 \%)$, ensino superior $(n=383 ; 78 \%)$, pós-graduação (n $=65 ; 13,2 \%)$ e dois $(0,4 \%)$ não forneceram a informação.

\section{Instrumentos}

Foi examinada a versão brasileira da Escala de Componentes do Amor-Reduzida (ECA-R) de Critelli et al. (1986) com 35 itens igualmente distribuídos em cinco subescalas: Dependência Romântica, Intimidade Comunicativa, Excitação Física, Respeito e Compatibilidade Romântica. Para registrar as respostas no instrumento, os participantes usaram uma escala tipo Likert de cinco pontos, de 1 (nada verdadeiro) a 5 (totalmente verdadeiro). Foi utilizada a versão brasileira do instrumento produzida por Hernandez e Oliveira (2003).

Com o objetivo de gerar evidências de validade convergente para a ECA-R, os participantes também responderam à Escala Triangular do Amor de SternbergReduzida (ETAS-R) produzida por Gouveia et al. (2009). $\mathrm{O}$ instrumento tem 15 itens igualmente distribuídos em três subescalas: Intimidade, Paixão e Decisão/Compromisso, que representam os três componentes do amor propostos pela teoria triangular do amor de Sternberg $(1986,1997)$. Gouveia et al. (2009) definiram os 15 itens da ETAS-R a partir dos resultados da primeira adaptação brasileira (Hernandez, 1999). Para o estudo atual, após uma revisão comparativa dos itens traduzidos da ETAS-R com as versões completas da ETAS (Sternberg, 1998), optou-se pela reformulação do item "Sonho de olhos aberto com............." por "Com frequência, durante o dia, me descubro pensando em..............". Sternberg (2006, p. 197) registrou que "A versão completa mais recente da Escala Triangular do Amor pode ser encontrada em Sternberg (1998)". A reformulação citada foi baseada na última versão do instrumento em inglês. Os participantes usaram uma escala tipo Likert de cinco pontos, de 1 (nada verdadeiro) a 5 (totalmente verdadeiro) para registrar as suas respostas.

\section{Coleta de Dados}

Após os participantes serem informados sobre os objetivos da pesquisa, assinaram o Termo de Consentimento Livre e Esclarecido, conforme as diretrizes éticas para pesquisa envolvendo seres humanos contidas na Resolução $\mathrm{CNS} \mathrm{n}^{\circ}$ 196/96. Este projeto foi aprovado pelo COEP-UERJ mediante Parecer 97/2011. Os dados foram coletados nas salas de aula durante o período letivo regular universitário, em cursos de graduação e pós-graduação, visando pessoas com maior idade e relações amorosas mais estáveis. Também foram abordados participantes em projetos universitários de extensão comunitária, em associações e grupos de casais diversos, buscando dados em setores variados da sociedade. Os instrumentos foram aplicados de forma coletiva ou individual, conforme a disponibilidade dos respondentes.

\section{Análise dos Dados}

Para verificar a estrutura da escala foram realizadas Análises Fatoriais Exploratórias (AFEs) usando o programa Factor 9.2 (Lorenzo-Seva \& Ferrando, 2007). Para algumas análises relativas aos itens e escalas foi usado o SPSS 20.

\section{Resultados}

Os 16 escores $(<1 \%)$ que os sujeitos omitiram nas respostas a variados itens da ECA-R foram repostos pela média da série. A adequação dos dados à análise fatorial foi verificada por meio do teste Kaiser-Meyer-Olkin, que forneceu um valor de 0,96 e do teste de esfericidade de Bartlett, que apresentou os valores, $\chi^{2}(528)=12.141,40$, $p<0,001$.

Na AFE, cinco dimensões com autovalores maiores do que 1,0 (Kaiser, 1960) foram extraídas dos escores da ECA-R usando o método Minimum Rank Factor Analysis (MRFA, Ten Berge \& Kiers, 1991) e a rotação Direct Promin. Contudo, o método Minimum Average Partial Test (MAP, Velicer, 1976) usado para auxiliar na retenção de fatores, recomendou uma solução de três fatores (primeiro, 6,43 
da variância comum explicada, segundo, 9,03, e terceiro, $5,04)$ que juntos foram responsáveis pela explicação de $20,49(73,50 \%)$ da variância comum total de 27,89. Os valores das médias das correlações parciais geradas no MAP foram para a primeira dimensão 0,02842, para a segunda,
0,01311 , terceira, 0,01233 e quarta, 0,01838 . No primeiro fator extraído, com cargas que variaram de muito boas a excelentes $(0,65-0,84)$, saturaram os sete itens designados para a Dependência Romântica e os itens 29, 34 e 35 da Compatibilidade Romântica. No segundo, com cargas de boas

Tabela 1. Matriz de primeira ordem rotada de cargas fatorial e comunalidades dos itens da ECA-R

\begin{tabular}{|c|c|c|c|c|}
\hline \multirow{2}{*}{ Item } & \multicolumn{3}{|c|}{ Fatores } & \multirow{2}{*}{ h2 } \\
\hline & 1 & 2 & 3 & \\
\hline 1.Meu relacionamento com............é mais importante para mim do que qualquer coisa. & 0,77 & 0,03 & 0,07 & 0,78 \\
\hline 2.Seria muito difícil para mim ficar sem ............. & 0,84 & $-0,03$ & 0,07 & 0,88 \\
\hline 3.Para mim..............é o único amor real. & 0,84 & $-0,04$ & 0,03 & 0,80 \\
\hline 4.Eu necessito de .............. & 0,69 & 0,03 & 0,18 & 0,79 \\
\hline 5.Minha amizade com.............é mais importante para mim do que qualquer coisa. & 0,65 & 0,12 & $-0,09$ & 0,71 \\
\hline 6.Uma das minhas principais preocupações é com o bem-estar de.............. & 0,70 & 0,13 & $-0,13$ & 0,63 \\
\hline 7.Se eu nunca estivesse com .............. me sentiria um (a) miserável. & 0,65 & $-0,01$ & 0,13 & 0,68 \\
\hline 8.Eu sinto que ............me entende bem. & 0,18 & 0,55 & 0,16 & 0,82 \\
\hline 9.............e eu temos um relacionamento muito sólido. & 0,41 & 0,56 & $-0,11$ & 0,92 \\
\hline 10............é alguém com quem eu realmente posso me comunicar. & 0,03 & 0,71 & 0,14 & 0,92 \\
\hline 11.Eu sinto que posso confiar em ............. sobre todas as coisas. & 0,27 & 0,66 & $-0,10$ & 0,80 \\
\hline 12............é a pessoa que eu mais gostaria de falar sobre um problema. & 0,31 & 0,50 & $-0,01$ & 0,75 \\
\hline 13.Eu percebo que é muito fácil ir adiante junto com ............. & 0,24 & 0,55 & 0,03 & 0,75 \\
\hline $14 \ldots \ldots \ldots \ldots . . . . e$ eu temos valores muito semelhantes. & 0,15 & 0,57 & 0,12 & 0,82 \\
\hline 15.Eu me sinto muito romântico (a) com ............... & 0,18 & 0,29 & 0,51 & 0,83 \\
\hline 16.Eu fico muito excitado (a) sexualmente quando beijo ............ & $-0,09$ & 0,15 & 0,80 & 0,82 \\
\hline 17.Meus sentimentos por .............são com frequência altamente apaixonados. & 0,17 & 0,20 & 0,63 & 0,91 \\
\hline 18.Passo boa parte do tempo pensando em ............. & 0,44 & $-0,04$ & 0,46 & 0,74 \\
\hline 19.Quando eu vejo ............., minha primeira reação é de excitação. & 0,01 & $-0,08$ & 0,83 & 0,86 \\
\hline $\begin{array}{l}\text { 20.Com frequência, noto que meu coração bate mais acelerado ou noto sinais de excitação } \\
\text { quando estou perto de ............. }\end{array}$ & 0,02 & $-0,08$ & 0,89 & 0,89 \\
\hline 21.Eu sou muito atraído (a) fisicamente por .............. & 0,06 & 0,05 & 0,75 & 0,73 \\
\hline 22.Em minha opinião ...............é uma pessoa excepcionalmente madura. & $-0,05$ & 0,72 & 0,12 & 0,80 \\
\hline 23.Eu penso que ..............é sempre uma pessoa bem adaptada. & $-0,02$ & 0,70 & 0,17 & 0,83 \\
\hline 24.Eu recomendaria, com segurança, ........... para um trabalho de responsabilidade. & $-0,12$ & 0,78 & $-0,04$ & 0,76 \\
\hline 25.Eu tenho grande confiança no bom senso de .............. & $-0,14$ & 0,95 & $-0,01$ & 1,00 \\
\hline 26.Eu sei que poderia contar com..............se precisasse de ajuda para qualquer coisa. & $-0,01$ & 0,85 & $-0,10$ & 0,86 \\
\hline 27.Eu penso que ..............é uma daquelas pessoas que rapidamente conquista respeito. & $-0,00$ & 0,78 & $-0,01$ & 0,88 \\
\hline 28.A maioria das pessoas reagem favoravelmente a ..............após um breve contato. & 0,07 & 0,65 & 0,00 & 0,70 \\
\hline 29.Já que eu estou com .............., a felicidade será inevitável. & 0,39 & 0,38 & 0,24 & 0,88 \\
\hline 30.Quando eu estou com............ nós sempre estamos com o mesmo humor. & 0,09 & 0,35 & 0,40 & 0,75 \\
\hline 31.............satisfaz totalmente minhas necessidades de amor, afeto, amizade e segurança. & 0,24 & 0,57 & 0,15 & 0,88 \\
\hline 32.............e eu não discordamos sobre assuntos importantes. & 0,14 & 0,36 & 0,16 & 0,64 \\
\hline 33.Eu penso que ............. e eu somos muito parecidos um com o outro. & 0,16 & 0,38 & 0,28 & 0,80 \\
\hline 34.Como eu tenho ..........., não me sinto atraído por outras pessoas do sexo oposto. & 0,38 & $-0,00$ & 0,23 & 0,56 \\
\hline 35.Eu jamais poderia odiar ............ & 0,38 & 0,19 & $-0,07$ & 0,69 \\
\hline Variância comum explicada & 6,43 & 9,03 & 5,04 & \\
\hline Porcentagem da variância explicada & 23,1 & 32,4 & 18,1 & \\
\hline
\end{tabular}

Nota. Método de Extração: Minimum Rank Factor Analysis. Método de Rotação: Direct Oblimin. As cargas fatoriais $<0,30$ foram excluídas. Fatores: 1 = Dependência Romântica (7 itens) + Compatibilidade Romântica (3 itens); 2 = Intimidade Comunicativa (7 itens) + Respeito (7 itens) + Compatibilidade Romântica (3 itens); 3 = Excitação Física (7 itens) + Compatibilidade Romântica (1 item). 
a excelentes $(0,50-0,71)$, saturaram os sete itens designados para a Intimidade Comunicativa, os sete itens do Respeito, com cargas de muito boas a excelentes $(0,65-0,95)$ e, também, os itens 31, 32 e 33 da Compatibilidade Romântica. No terceiro fator extraído, com cargas de razoáveis a excelentes (0,46-0,89), carregaram os itens da Excitação Física e o item 30 da Compatibilidade Romântica. Observou-se que apenas os itens que deveriam pertencer à Compatibilidade Romântica saturaram de maneira dispersa nos três fatores extraídos.

Tabela 2. Resultados da Solução de Schmid-Leiman

\begin{tabular}{|c|c|c|c|c|}
\hline \multirow[t]{2}{*}{ Item } & \multirow{2}{*}{$\begin{array}{c}\begin{array}{c}\text { Fator de segunda } \\
\text { ordem }\end{array} \\
\text { G1 } \\
\end{array}$} & \multicolumn{3}{|c|}{$\begin{array}{c}\text { Fatores de primeira } \\
\text { ordem }\end{array}$} \\
\hline & & F1 & F2 & F3 \\
\hline 1 & 0,74 & 0,36 & 0,02 & 0,05 \\
\hline 2 & 0,77 & 0,40 & $-0,02$ & 0,05 \\
\hline 3 & 0,74 & 0,40 & $-0,03$ & 0,02 \\
\hline 4 & 0,74 & 0,32 & 0,02 & 0,14 \\
\hline 5 & 0,59 & 0,30 & 0,09 & $-0,07$ \\
\hline 6 & 0,62 & 0,33 & 0,10 & $-0,10$ \\
\hline 7 & 0,65 & 0,30 & $-0,01$ & 0,10 \\
\hline 8 & 0,62 & 0,08 & 0,41 & 0,12 \\
\hline 9 & 0,67 & 0,19 & 0,42 & $-0,09$ \\
\hline 10 & 0,58 & 0,01 & 0,53 & 0,11 \\
\hline 11 & 0,61 & 0,12 & 0,50 & $-0,08$ \\
\hline 12 & 0,60 & 0,15 & 0,38 & $-0,01$ \\
\hline 13 & 0,60 & 0,11 & 0,41 & 0,03 \\
\hline 14 & 0,59 & 0,07 & 0,43 & 0,10 \\
\hline 15 & 0,67 & 0,08 & 0,22 & 0,39 \\
\hline 16 & 0,53 & $-0,04$ & 0,12 & 0,62 \\
\hline 17 & 0,68 & 0,08 & 0,15 & 0,49 \\
\hline 18 & 0,65 & 0,21 & $-0,03$ & 0,35 \\
\hline 19 & 0,49 & 0,01 & $-0,06$ & 0,65 \\
\hline 20 & 0,52 & 0,01 & $-0,06$ & 0,69 \\
\hline 21 & 0,56 & 0,03 & 0,04 & 0,58 \\
\hline 22 & 0,50 & $-0,03$ & 0,54 & 0,09 \\
\hline 23 & 0,55 & $-0,01$ & 0,53 & 0,13 \\
\hline 24 & 0,39 & $-0,05$ & 0,58 & $-0,03$ \\
\hline 25 & 0,51 & $-0,06$ & 0,71 & $-0,01$ \\
\hline 26 & 0,49 & $-0,01$ & 0,64 & $-0,01$ \\
\hline 27 & 0,51 & $-0,00$ & 0,59 & $-0,01$ \\
\hline 28 & 0,49 & 0,03 & 0,48 & 0,00 \\
\hline 29 & 0,75 & 0,18 & 0,29 & 0,18 \\
\hline 30 & 0,57 & 0,04 & 0,26 & 0,31 \\
\hline 31 & 0,68 & 0,11 & 0,43 & 0,12 \\
\hline 32 & 0,46 & 0,05 & 0,27 & 0,12 \\
\hline 33 & 0,58 & 0,07 & 0,29 & 0,22 \\
\hline 34 & 0,48 & 0,18 & $-0,00$ & 0,18 \\
\hline 35 & 0,42 & 0,18 & 0,14 & $-0,05$ \\
\hline \multicolumn{5}{|c|}{$\begin{array}{c}\text { Solução fatorial de } \\
\text { segunda ordem }\end{array}$} \\
\hline G1 & & 0,88 & 0,66 & 0,63 \\
\hline
\end{tabular}

Os índices de simplicidade fatorial de Bentler (1977) e Lorenzo-Seva (2003) foram, respectivamente, 0,99 e 0,44. Estes sugeriram que a solução geral encontrada apresentou simplicidade ou parcimônia. Para facilitar a interpretação da existência de uma dimensão geral na ECA-R compreendendo os três fatores extraídos na AFE, também foi calculada uma solução fatorial de segunda ordem (Tabela 2), por meio da Solução de Schmid-Leiman (SSL) de Schmid e Leiman (1957).

As correlações entre os três fatores foram moderadas variando de 0,42 a 0,58. Nas Tabelas 3 e 4 são apresentados os valores das estatísticas descritivas, das correlações entre os itens e item-total para os três dimensões e para a dimensão geral (ECA-R).

O Coeficiente de Correlação de Pearson apurou correlações estatísticas significativas $(p<0,001)$ entre os fatores da ECA-R, os fatores da ETAS-R e as duas escalas totais. Os coeficientes variaram de moderados, moderados fortes e fortes (Tabela 5).

Como a estrutura fatorial da ECA-R ficou muito semelhante ao modelo da ETAS-R, com três fatores, uma nova AFE, com o mesmo método, foi realizada juntando os escores de ambas as escalas, totalizando 50 itens. Nos resultados, seis dimensões com autovalores maiores do que 1,0 (Kaiser, 1960) foram extraídas. No entanto, o método MAP recomendou uma solução de três fatores, que juntos foram responsáveis pela explicação de 28,5 (69,37\%) da variância comum total de 41,09. As médias das correlações parciais foram as seguintes: 0,02622 (fator 1), 0,01026 (fator 2) e 0,00810 (fator 3 ).

No primeiro fator extraído, com 9,42 (23\%) da variância comum explicada, carregaram os sete itens da Dependência Romântica, dois itens (29 e 34) da Compatibilidade Romântica (ambos componentes da ECA-R) e os cinco itens do componente Compromisso da ETAS-R. No segundo fator, com 11,94 (29\%) da variância comum explicada, os sete itens da Intimidade Comunicativa, os sete itens do Respeito, quatro itens (30, 31, 32 e 33) da Compatibilidade Romântica (componentes da ECA-R) e os cinco itens do componente Intimidade da ETAS-R. No terceiro, com 7,13 (17\%) da variância comum explicada, os sete itens da Excitação Física da ECA-R e os cinco itens da Paixão da ETAS-R. Nessa solução, o item 35 da ECA-R não apresentou carga fatorial mínima necessária $(>0,30)$, ou seja, sem significância prática e, portanto, seria candidato a exclusão. Os indicativos de simplicidade e parcimônia para a solução (Bentler, 1977; Lorenzo-Seva, 2003) foi de 0,99 e da existência de uma dimensão geral (Schmid \& Leiman, 1957) mantiveram-se com melhorias.

\section{Discussão}

Os dois únicos estudos psicométricos encontrados na literatura examinaram a ECA-R (Carreño \& Serrano, 1995; Critelli et al., 1986) por meio de Análise de Componentes Principais com rotação varimax e o critério de Kaiser (1960) para a retenção de cinco fatores. Ambos os estudos reconheceram as relações entre as subescalas da ECA-R, representadas por moderados a fortes coeficientes de 
Tabela 3. Matriz de Correlação dos Itens da ECA-R

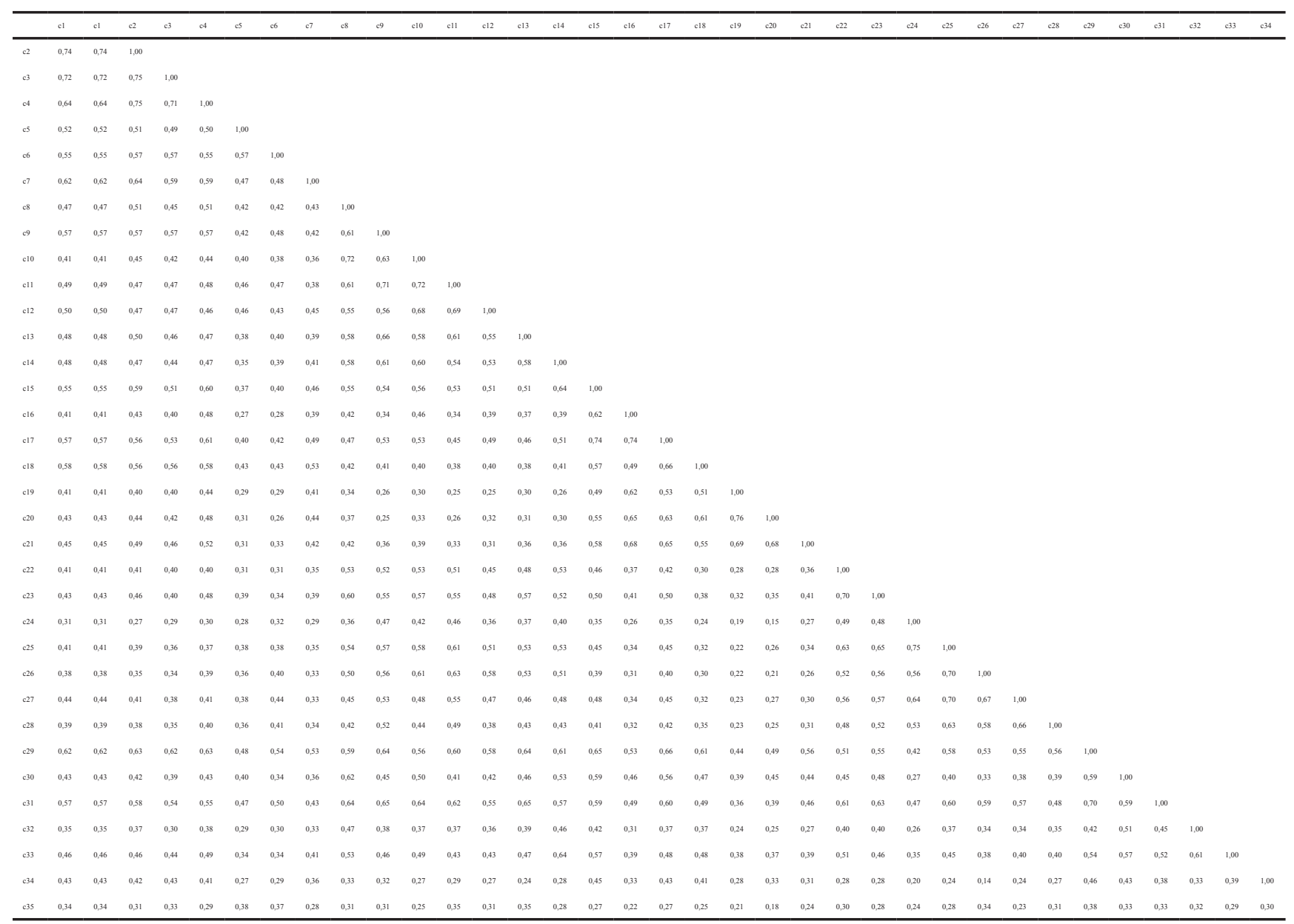

Nota. Todas as correlações foram significativas no nível 0,01 (1 extremidade)

Tabela 4. Estatísticas Descritivas, Coeficientes Alfas de Cronbach, Correlações Inter-itens e Itens-total

\begin{tabular}{ccccccc}
\hline Fatores & Médias & DP & Alfas de Cronbach & Correlação inter-item & Correlação item-total \\
\hline 1 & 35,2 & 9,17 & 0,91 & $r_{\text {médio }}=0,50(0,27$ a 0,75$)$ & 0,43 a 0,80 \\
2 & 66,3 & 14,41 & 0,95 & $r_{\text {médio }}=0,53(0,26$ a 0,75$)$ & 0,60 a 0,78 \\
3 & 27,4 & 7,50 & 0,92 & $r_{\text {médio }}=0,58(0,39$ a 0,76$)$ & 0,58 a 0,81 \\
ECA-R total & 128,9 & 27,96 & 0,97 & $r_{\text {médio }}=0,45(0,14$ a 0,76$)$ & 0,43 a 0,83 \\
\hline
\end{tabular}

Tabela 5. Coeficientes de Correlação de Pearson entre os Componentes da ECA-R e da ETAS-R

\begin{tabular}{|c|c|c|c|c|c|c|c|}
\hline Dimensões & Fator 1 & Fator 2 & Fator 3 & ECA-R & Compromisso & Paixão & Intimidade \\
\hline Fator 2 & 0,73 & & & & & & \\
\hline Fator 3 & 0,72 & 0,65 & & & & & \\
\hline ECA-R & 0,90 & 0,93 & 0,84 & & & & \\
\hline Compromisso & 0,84 & 0,70 & 0,65 & 0,81 & & & \\
\hline Paixão & 0,60 & 0,57 & 0,83 & 0,72 & 0,62 & & \\
\hline Intimidade & 0,61 & 0,82 & 0,55 & 0,77 & 0,60 & 0,55 & \\
\hline ETAS-R & 0,82 & 0,81 & 0,79 & 0,90 & 0,90 & 0,85 & 0,82 \\
\hline
\end{tabular}


correlação. Em decorrência disso, estes pesquisadores consideraram a ECA-R com cinco dimensões diferentes e claramente estruturadas ligadas a uma dimensão geral, o amor.

O estudo atual buscou a identificação de dimensões ou construtos latentes representados pelas variáveis ou itens (Hair, Black, Babin, Anderson, \& Tatham, 2009). Foram utilizadas técnicas estatísticas consideradas mais adequadas para os objetivos propostos do que fizeram os estudos anteriores (Carreño \& Serrano, 1995; Critelli et al., 1986). O método MRFA com rotação Direct Promin extraiu os cinco fatores do modelo original baseado em autovalores (Kaiser, 1960), critério que tem se caracterizado pela tendência em superestimar o número de fatores extraídos (Damásio, 2012; Laros, 2005). Por outro lado, uma solução de três fatores foi recomendada pelo MAP, apresentando os seguintes valores de correlações médias parciais: $0,02622,0,01026,0,00810$ e 0,00969 .

Desta forma, o resultado do presente estudo não corroborou a estrutura fatorial original da ECA-R postulada por Critelli et al. (1986) e replicada por Carreño e Serrano (1995). Possivelmente, os diferentes métodos de análise de dados são responsáveis pelos resultados diversos.

$\mathrm{Na}$ presente AFE, todos os itens apresentaram cargas fatoriais maiores do que $0,30 \mathrm{em}$ pelo menos um dos três fatores extraídos. As correlações entre os fatores foram todas acima de 0,40 , indicando que os mesmos são relacionados, $o$ que foi confirmado pela análise fatorial de segunda ordem na qual é obtido um fator geral. Todos os três fatores de primeira ordem saturaram mais do que 0,60 no fator geral. Isso sugere a existência de um fator geral (amor), além dos três fatores de primeira ordem. O fator geral subjacente, possivelmente, compreenderá, pelo menos em parte, o que seja o amor.

Os Coeficientes de Correlação de Person mostraram correlações de moderadas fortes a fortes entre todas as dimensões da ECA-R e da ETAS-R. A nova AFE indicou que esses dois conjuntos de itens, da ECA-R e da ETAS-R, podem se ajustar com nitidez ao modelo proposto por Sternberg (1988) na Teoria Triangular do Amor, dos três componentes do amor (Paixão, Intimidade e Decisão/Compromisso). A observação da distribuição dos componentes de Critelli et al. (1986) e de Sternberg (1998) nos três fatores extraídos e a análise de conteúdo dos itens carregados em cada destes indicaram que o novo arranjo estrutural da ECA-R se aproximou muito da estrutura da ETAS-R.

Segundo Critelli et al. (1986), os itens da Dependência Romântica são caracterizados pela crença nos ideais românticos, na ênfase da importância do relacionamento, na necessidade do parceiro e na exclusividade do amor. $\mathrm{Na}$ construção desse componente houve a influência da concepção de amor romântico de Rubin (1973), que também influenciou o construto Decisão/Compromisso (Sternberg, 1988). Os conteúdos dos itens que compõem essa dimensão refletem, em geral, o reconhecimento da importância do amor e a dedicação para mantê-lo.

A Intimidade Comunicativa é o componente que enfatiza o sentimento de ser compreendido pelo outro, de participar de um relacionamento sólido, havendo comunicação e confiança no parceiro (Critelli et al., 1986). De modo similar, a Intimidade, segundo Sternberg (1988), é o elo que une o casal mediante dedicação e afeição recíprocas. A Intimidade está presente nos relacionamentos que possuem: desejo de promover o bem-estar da outra pessoa; felicidade de compartilhar experiências de vida; senso mútuo de respeito; capacidade de contar com a pessoa amada em momentos de necessidade; compreensão mútua da presença do outro nos momentos difíceis da vida; compreensão íntima compartilhada de sintonia no mesmo comprimento de onda; disposição para apoiar um ao outro; disponibilidade para receber o apoio emocional do outro; capacidade de comunicação além dos níveis superficiais ou práticos; e, reconhecimento mútuo do valor do parceiro na sua vida. Os itens do componente Respeito da ECA-R também aderiram a este fator composto pela Intimidade Comunicativa (ECA-R) e a Intimidade (ETAS-R). O respeito mútuo é um dos aspectos que compõe o conceito de intimidade de Sternberg (1988). Para identificar e delimitar esses construtos, tanto Sternberg e Grajek (1984) quanto Critelli et al. (1986) inspiraram-se nas escalas de amar e gostar de Rubin (1970).

No fator 3, agruparam-se os itens da Excitação Física da ECA-R e os da Paixão da ETAS-R, componente que se refere ao estado romântico e sexualmente excitado, apaixonado e fisicamente atraído pelo outro e, portanto, relacionado com necessidades de ordem primária (Critelli et al., 1986). Os itens da Paixão de Sternberg (1988) referem-se aos impulsos que levam ao romance, à atração física, à consumação sexual e a outros fenômenos relativos ao amor.

O comportamento dispersivo dos itens da Compatibilidade Romântica foi observado por Carreño e Serrano (1995). Critelli et al. (1986) reconheceram que esse componente combinaria uma mistura de aspectos de diversos componentes do amor, tais como, a semelhança de humor, a interação harmoniosa, a satisfação total de necessidade e o contentamento. No estudo atual, essa condição está representada no desempenho confuso que esses itens apresentaram no estudo atual. Contudo, pode-se constatar a razoável compatibilidade dos mesmos aos construtos que aderiram, apesar das cargas fatoriais mais fracas.

Adicionalmente, a SSL, que é uma transformação das matrizes de cargas fatoriais obtidas a partir da análise fatorial, forneceu informações complementares revelando, de forma independente, a influência dos fatores de primeira ordem e dos fatores de ordem superior sobre as variáveis observadas. $\mathrm{Na}$ Tabela 2 podem ser observadas essas relações diretas (cargas fatoriais) entre os fatores de primeira e segunda ordem e as variáveis (itens) observadas. Ou seja, estão sendo apresentadas as cargas fatoriais exclusivas de cada um dos itens em cada um dos fatores de primeira ordem e de segunda ordem.

As cargas dos fatores de primeira ordem da ECA são menores (Tabela 2) do que as cargas originais produzidas na análise fatorial de primeira ordem (Tabela 1), porque na SSL as cargas de primeira ordem foram reduzidas a uma parte específica, a outra parte carregou no fator geral de segunda ordem. Nessa perspectiva, embora a estrutura de fatores de primeira ordem tenha se mantido, a maior parte dos itens (27), simultânea e independentemente, apresentou cargas mais fortes no G1 do que nos F1, F2 e F3. Destes, os itens $29,32,33,34$ e 35 apresentaram cargas fatoriais menores do que 0,30 , embora nas cargas originais (Tabela 1 ) as mesmas 
estavam acima desse mínimo. Nesses casos, esses itens não se apresentam como uma "boa" medida das dimensões específicas, mas refletem mais a dimensão geral. Todos esses itens originalmente pertencem à dimensão Compatibilidade Romântica da ECA que foi reconhecida por Critelli et al. (1986) com um misto de diversos aspectos do amor. De outra forma, oito itens saturaram mais forte nas dimensões específicas (F2 e F3) do que na dimensão geral (Tabela 2). Esses representam uma medida mais pura e específica das dimensões que representam.

Problemas de dispersão e superposição de cargas fatoriais dos itens entre diversos fatores foram encontrados nas adaptações brasileiras da Escala Triangular do Amor de Sternberg (1997) por Hernandez (1999) e Cassep-Borges e Teodoro (2007). Em tentativas de superação dessa falta de clareza fatorial é que foram propostas versões reduzidas do instrumento (Cassep-Borges \& Teodoro, 2009; Gouveia et al., 2009). Na análise atual da ECA-R, ainda que a estrutura fatorial original de cinco fatores não tenha sido encontrada, os fatores se apresentaram bem definidos com os seus itens saturando fortes em uma única dimensão (exceto os itens da Compatibilidade Romântica) e muito raramente apresentando cargas fatoriais significativas simultâneas em outras dimensões. O instrumento de Critelli et al. (1986) embora reduzido, possui 35 itens, futuras análises dos mesmos poderão dar subsídios para o aperfeiçoamento das medidas do amor.

As evidências de similaridade entre as propostas de Critelli et al. (1986) e Sternberg (1986) indicam que, no geral, os itens da ECA-R e da ETAS-R representam os mesmos construtos. Esses resultados poderão contribuir para a busca da definição e mensuração do amor, conceito que ainda carece de clareza na Psicologia. Entretanto, os mesmos devem ser ponderados considerando suas limitações. Por exemplo, a amostra usada é de conveniência e restrita ao sul do País, pode não refletir a diversidade cultural da população brasileira. Por outro lado, não era totalmente constituída por universitários solteiros (Carreño \& Serrano, 1995; Critelli et al., 1986), mais de um terço dos participantes estavam envolvidos em relacionamentos mais sérios e coabitavam com seus parceiros. Entretanto, sugere-se que novas investigações com amostras probabilísticas e mais abrangentes possam preencher essa lacuna.

\section{Referências}

Bentler, P. M. (1977). Factor simplicity index and transformations. Psychometrika, 59, 567-579.

Berscheidt, E. (2010). Love in the fourth dimension. Annual Review of Psychology, 61, 1-25. doi: 10.1146/annurev. psych.093008.100318

Carreño, M., \& Serrano, G. (1995). Análisis de instrumentos para la medida del amor. Revista de Psicologia Social, 10, 131-148.

Cassep-Borges, V., \& Teodoro, M. (2007). Propriedades Psicométricas da Versão Brasileira da Escala Triangular do Amor de Sternberg. Psicologia: Reflexão e Crítica, 20, 513522.
Cassep-Borges, V., \& Teodoro, M. (2009). Versión Reducida de la Escala Triangular del Amor: Características del Sentimiento en Brasil. Interamerican Journal of Psychology, 43, 30-38.

Critelli, J. W., Myers, E. J., \& Loos, V. E. (1986). The components of love: Romantic attraction and sex role orientation. Journal of Personality, 54, 355-370. doi: 10.1111/j.1467-6494.1986. tb00399. $\mathrm{x}$

Damásio, B. F. (2012). Uso da análise fatorial exploratória em psicologia. Avaliação Psicológica, 11, 213-228.

Driscoll, R., Davis, K., \& Lipetz, M. (1972). Parental interference and romantic love. Journal of Personality and Social Psychology, 24, 1-10.

Fromm, E. (1966). A arte de amar. Belo Horizonte: Itatiaia. (Trabalho original publicado em 1956)

Gouveia, V. V., Fonseca, P. N., Cavalcanti, J. P. N., Diniz, P. K. C., \& Dória, L. C. (2009). Versão abreviada da Escala Triangular do Amor: evidências de validade fatorial e consistência interna. Estudos de Psicologia (Natal), 14, 31-39. doi:10.1590/S1413294X2009000100005

Graham, J. M., \& Christiansen, K. (2009). The reliability of romantic love: A reliability generalization meta-analysis. Personal Relationships, 16, 49-66. doi: 10.1111/j.14756811.2009.01209.x

Hair Jr., J. F., Black , W. C., Babin, B. J., Anderson, R. E., \& Tatham, R. L. (2009). Análise Multivariada de Dados. Porto Alegre: Bookman.

Hatfield, E., Bensman, L., \&. Rapson, R. L . (2011). A brief history of social scientists' attempts to measure passionate love. Journal of Social and Personal Relationships, 29, 143-164. doi: 10.1177/0265407511431055

Hatfield, E., \& Sprecher, S. (1986). Measuring passionate love in intimate relationships. Journal of Adolescence, 9, 383-410. doi:10.1016/S0140-1971(86)80043-4

Hendrick, C., \& Hendrick, S. (1986). A theory and method of love. Journal of Personality and Social Psychology, 50, 392-402. doi:10.1037/0022-3514.50.2.392

Hernandez, J. A. E. (1999). Validação da Estrutura da Escala Triangular do Amor: Análise Fatorial Confirmatória. Aletheia, 9, 15-26.

Hernandez, J. A. E., \& Oliveira, I. M. B. (2003). Os componentes do amor e a satisfação. Psicologia: Ciência e Profissão, 21, 58-69.

Kaiser, H. F. (1960). The application of eletronic-computers to fator-analysis. Educational and Psychological Measurement, 20, 141-151. doi: 10.1177/001316446002000116

Laros, J. A. (2005). O uso da análise fatorial: algumas diretrizes para pesquisadores. In L. Pasquali (Ed..), Análise fatorial para pesquisadores (pp. 163-184). Brasília: LabPAM.

Lorenzo-Seva, U. (2003). A factor simplicity index. Psychometrika, $68,49-60$.

Lorenzo-Seva, U., \& Ferrando, P. J. (2006). FACTOR: A computer program to fit the Exploratory Factor Analysis model. Behavior Research Methods, Instruments \& Computers, 38, 88-91.

Maslow, A. H. (1974). Introdução à psicologia do ser. Rio de Janeiro: Eldorado. (Trabalho original publicado em 1968)

Masuda, M. (2003). Meta-analyses of love scales: Do various love scales measure the same psychological constructs. Japanese Psychological Research, 45, 25-37. doi: 10.1111/14685884.00030

Rubin, Z. (1970). Measurement of romantic love. Journal of Personality and Social Psychology, 16, 265-273. 
Rubin, Z. (1973) Liking and Loving. An invitation to Social Psychology. New York: Holt, Rinehart and Winston, Inc.

Schmid, J., \& Leiman, J. N. (1957). The development of hierarchical factor solutions. Psychometrika, 22, 53-61.

Sternberg, R. J. (1986). A triangular theory of love. Psychological Review, 93, 119-135. doi:10.1037/0033-295X.93.2.119

Sternberg, R. J. (1988). The Triangle of Love. New York: Basic Books.

Sternberg, R. J. (1997). Construct validation of a triangular love scale. European Journal of Psychology, 27, 313-335. doi:10.1002/(SICI)1099-0992(199705)27:3<313::AIDEJSP824>3.0.CO;2-4
Sternberg, R. J. (1998). Cupid's arrow: The course of love through time. Cambridge: Cambridge University Press.

Sternberg, R. J. (2006). A duplex theory of love. In R. J. Sternberg \& K. Weis (Eds.), The New Psychology of Love (pp.184-199). New Haven: Yale University Press.

Sternberg, R. J., \& Grajek, S. (1984). The nature of love. Journal of Personality and Social Psychology, 47, 312-329.

Ten Berge, J. M. F., \& Kiers, H. A. L. (1991). A numerical approach to the approximate and the exact minimum rank of a covariance matrix. Psychometrika, 56, 309-315.

Velicer, W. F. (1976). Determining the number of components from the matrix of partial correlations. Psychometrika, 41, 321-327. 